\title{
OPTIMAL POLYNOMIAL BOUNDS FOR THE EXPONENTIAL FUNCTION
}

\author{
JAEGUG BAE
}

Abstract. We find polynomial lower and upper bounds of $e^{x}$ on some respective intervals. To be specific, for each natural number $n$, we construct polynomials $p_{n}(x)$ and $q_{n}(x)$ of degree $n$ so that $p_{n}(x) \leqslant p_{n+1}(x) \leqslant e^{x}$ and $e^{x} \leqslant q_{n+1}(x) \leqslant q_{n}(x)$ on some intervals, respectively. These polynomials are optimal in the sense that if $p(x)$ (or $q(x))$ is a polynomial of degree $n$ with $p_{n-1}(x) \leqslant p(x) \leqslant e^{x}$ (or $\left.e^{x} \leqslant q(x) \leqslant q_{n-1}(x)\right)$ then $p(x) \leqslant p_{n}(x)$ (or $q_{n}(x) \leqslant$ $q(x))$. The fact that $1 / p_{n}(-x)$ works as an upper bound of $e^{x}$ on a switched interval is interesting. We also provide the size comparison between two upper bounds $q_{n}(x)$ and $1 / p_{n}(-x)$.

Mathematics subject classification (2010): Primary 33B10; secondary 11A99.

Keywords and phrases: Bounds, polynomials, the exponential function.

\section{REFERENCES}

[1] J. BAE, Optimal polynomial lower bounds for the exponential function, Honam Math. J. 29, 4 (2007), $535-542$

[2] J. BAE, S. Kim, On a generalization of an upper bound for the exponential function , J. of Math. Anal. and Appl. 353, 1 (2009), 1-7.

[3] J. Karamata, Sur l'approximation de $e^{x}$ par des fonctions rationnelles (in Serbian), Bull. Soc. Math. Phys. Serbie 1 (1949), 7-19.

[4] S. KIm, Densely algebraic bounds for the exponential function, Proc. Amer. Math. Soc. 135 (2007), 237-241.

[5] D. S. Mitrinovic, Analytic Inequalities, Springer-Verlag, New York, 1970.

[6] W. E. SEwelL, Some inequalities connected with exponential function (in Spanish), Rev. Ci (Lima) 40 (1938), 453-456. 